\title{
Mass transfer and thermodynamics during immersion precipitation for a two-polymer system Evaluation with the system PES-PVP-NMP-water
}

\author{
R.M. Boom*, Th. van den Boomgaard, C.A. Smolders \\ University of Twente, Faculty of Chemical Technology, P.O. Box 217, 7500 AE Enschede, Netherlands
}

(Received September 29, 1992; accepted in revised form January 10, 1994)

\begin{abstract}
An extended version of the mass transfer model by Reuvers et al. for a four-component system is evaluated, which is shown to be generally valid for short times. The thermodynamics under these circumstances are evaluated, together with the kinetics. Initial composition paths (concentration profiles) are calculated. It appears that delay of demixing is not possible when a polymeric additive is used, which is soluble in the nonsolvent, while the velocity of demixing decreases. The calculations are evaluated for the system poly(ether sulfone)poly (vinylpyrrolidone) $-N$-methylpyrrolidone-water by means of light transmission measurements during immersion precipitation, for a wide range of compositions of the polymer solution and coagulation bath.
\end{abstract}

Key words: Membrane preparation; Phase inversion; Immersion precipitation; Diffusion; Polymer blend

\section{Introduction}

The immersion precipitation technique [1] is very suitable for preparation of asymmetric membranes with properties varying from microfiltration to gas separation. The most simple system to perform immersion precipitation is a (quasi-) ternary system consisting of a polymer, a solvent and a nonsolvent.

The immersion precipitation process is governed by the thermodynamic and diffusional properties of the components present in the system. The thermodynamics underlying mem-

\footnotetext{
"Corresponding author. Current address: Unilever Research Laboratory Vlaardingen, P.O. Box 114, 3130 AC Vlaardingen, Netherlands.
}

brane formation in such a ternary system have already been studied intensively by, e.g., Tompa [2], Hsu and Prauznitz [3], and Altena and Smolders [4], all based on the Flory-Huggins theory. A study of the kinetic basis of the immersion precipitation process was started by Cohen et al. [6]. Reuvers et al. [7,8] further developed this model; they showed that the assumptions on cross-diffusional coefficients that Cohen et al. made could not be justified.

Reuvers et al. [7,8] was able, using binary data on thermodynamics and on the diffusional behavior of the components, to predict some important characteristics of membrane formation by immersion precipitation. Especially the two types of demixing (and therefore of membrane formation ), that can be distinguished for a ter- 
nary membrane-forming system, could be adequately predicted delayed demixing and instantaneous demixing. Smolders et al. [9] found that the occurrence of large finger-like cavities (usually called macrovoids) are connected with the instantaneous demixing mechanism. They proposed a mechanism for the formation of macrovoids based on these two demixing regimes, which is in agreement with all experimental data known to the authors.

The mass transfer model by Reuvers et al. $[7,8]$ is meant as an approximation for the time span that the polymer solution film can still be regarded as being infinitely thick. In recent years, McHugh et al. [10-12] have further developed the mass transfer model by Reuvers et al. to describe the solvent/nonsolvent exchange process not only in the first moments, but until the onset of the demixing process. In our case, we restrict ourselves to the initial composition path (vide infra). Therefore, the extra computational effort for the approach by $\mathrm{McHugh}$ et al. is not useful for us. We will restrict ourselves to the approach by Reuvers et al. [7,8].

Understanding of the processes taking place in a ternary system is absolutely essential as a basis for comprehending the membrane formation mechanism. In practice, membranes are, however, never made from only three components. Extra components (additives) are used, which can result in a far greater variety of membrane structures than can be explained with a ternary model. As an example, a weak nonsolvent may be added to the polymer solution. This causes the polymer solution to become less compatible with the coagulation bath. Demixing is faster and macrovoid formation, for example, may be suppressed [13]. In the past decades, several authors [14-17] reported on the use of a nonsolvent (water) soluble polymeric additive to the polymer solution, which suppressed macrovoid formation and increased pore interconnectivity. Higher porosities and a modification of the surface properties of the membrane pores could be obtained. A typical system is poly (ether sulfone) (PES) as membrane-forming polymer, poly(vinyl-pyrrolidone) (PVP) as polymeric additive, and $N$-methylpyrrolidone and water as solvent and coagulation medium.

A real understanding of the effects of such an additive is not yet available. Cabasso et al. [18] suggested that PVP not really mixes with the polymer used and therefore created "islands" of PVP in a membrane-forming polymer matrix. Roesink [17] suggested that during immersion in the coagulation bath, the PVP molecules started to diffuse out of the polymer solution, into the nuclei formed, and became trapped on their way out, due to the extremely low diffusivities in a concentrated polymer solution. Although this approach explained the fact that PVP can never be completely removed from the membrane by rinsing, it could not explain other effects, such as the effect of PVP addition on macrovoid formation.

In our opinion, a quaternary membrane-forming system consisting of a nonsolvent, a solvent, a membrane forming polymer and a polymeric additive, is too complicated to be approached without a fundamental basis. In this paper we will further develop a mass transfer model for a four component system that was presented earlier in a basic form by the authors [19].

The characteristics of this model and the effect of these characteristics on the formation of membranes from a solution with two polymers in the casting solution will be evaluated here.

\section{Theory}

The mass transfer model, based on the ternary model by Reuvers et al. [7,8] and extended for a quaternary system is summarized in Table 1 . For the derivations and details we refer to Reuvers et al. [7] and to earlier work [19]. The conventions concerning the coordinates are shown in Fig. 1.

To find expressions for the phenomenological coefficients $L_{i j}$, the Maxwell-Stefan approach $[7,8,21]$ is used:

$\frac{\partial \mu_{i}}{\partial x}=\sum_{j=1}^{4} c_{j} R_{i j}\left(v_{j j}-v_{i}\right) \quad i=1, \ldots, 4$

where $R_{i j}$ is the friction coefficient between com- 
Table 1

Summary of the model as presented earlier [19], based on the ternary model by Reuvers et al. [7]

\begin{tabular}{|c|c|c|c|}
\hline \multicolumn{2}{|c|}{ Polymer solution } & \multirow[t]{2}{*}{ Interface } & Coagulation bath \\
\hline $\begin{array}{l}\text { Diffusion } \\
\text { equation }\end{array}$ & $\frac{\partial\left(\frac{\phi_{i}}{\phi_{3}}\right)}{\partial t}=\bar{v}_{i} \frac{\partial}{\partial m}\left\{\sum_{j=1,2,4} \phi_{3} L_{i j} \frac{\partial \mu_{j}}{\partial m}\right\}$ & & $\begin{aligned} \frac{\partial \phi_{i}}{\partial t} & =\bar{v}_{i} \frac{\partial}{\partial y}\left\{\sum_{j=1,2,4} L_{i j} \frac{\partial \mu_{j}}{\partial m}\right\} \\
& -\frac{\partial \phi_{i}}{\partial y}\left\{\sum_{k=1,2,4} J_{k}^{\chi=0}\right\}\end{aligned}$ \\
\hline $\begin{array}{l}\text { Spatial } \\
\text { coordinate }\end{array}$ & $\begin{array}{l}m=\int_{0}^{x} \phi_{3} \mathrm{~d} \xi \\
x: \text { distance from interface } \\
\text { into the polymer solution }\end{array}$ & & $\begin{array}{l}y=-x+X(t) \\
y: \text { distance from interface } \\
\text { into the coagulation bath }\end{array}$ \\
\hline $\begin{array}{l}\text { Boundary } \\
\text { conditions }\end{array}$ & $\left(\frac{\partial \phi_{i}}{\partial t}\right)^{m=\infty}=0$ & $J_{i}^{m=0}=J_{i}^{y=0}$ & $\left(\frac{\partial \phi_{i}}{\partial t}\right)^{p=\infty}=0$ \\
\hline
\end{tabular}
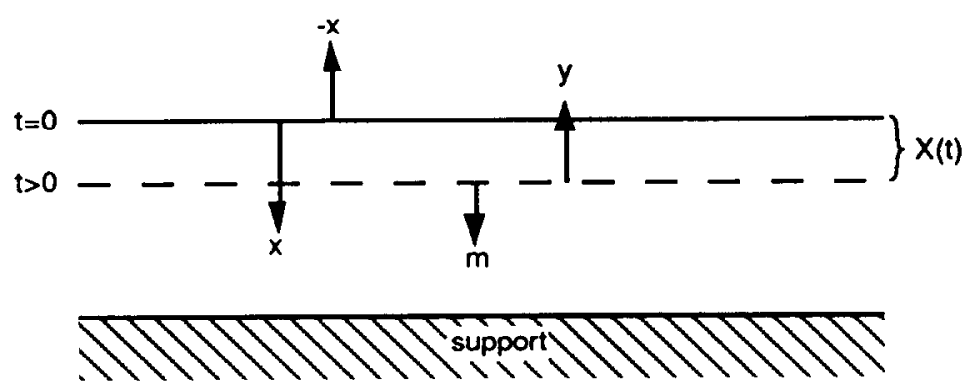

Fig. 1. The coordinates in the polymer solution and in the coagulation bath. After Reuvers et al. [7].

ponents $i$ and $j ; c_{i}$ is the concentration of component $i$ (defined as $\phi_{i} / \bar{v}_{i}$ ), and $v_{i}$ is the velocity in laboratory coordinates (see also Reuvers et al. [7]).

From this, it can be derived that the driving forces $X$ (united in the vector $X$ ) are related to the friction coefficients by:

$\mathbf{X}=\mathbf{R}\left[\begin{array}{l}c_{1}\left(v_{1}-v_{3}\right) \\ c_{2}\left(v_{2}-v_{3}\right) \\ c_{4}\left(v_{4}-v_{3}\right)\end{array}\right]$

in which

$X_{i}=\frac{\partial \mu_{i}}{\partial x}$

and the $3 \times 3$-matrix $\mathbf{R}$ is:

$$
\left.\begin{array}{c}
-\frac{1}{c_{1}}\left(c_{2} R_{12}+c_{3} R_{13}+c_{4} R_{14}\right) \\
R_{12} \\
R_{14} \\
\\
-\frac{1}{c_{2}}\left(c_{1} R_{12}+c_{3} R_{23}+c_{4} R_{24}\right) \\
R_{24} \\
\\
R_{14} \\
R_{24} \\
-\frac{1}{c_{4}}\left(c_{1} R_{14}+c_{2} R_{24}+c_{3} R_{34}\right)
\end{array}\right]
$$

in which the Onsager reciprocal relations are assumed to be valid $\left(R_{i j}=R_{j i}\right)$. In this terminol- 
ogy, the $3 \times 3$-matrix of phenomenological coefficients $L_{i j}$, $\mathbf{L}$ is determined by:

$\mathbf{L}=\mathbf{R}^{-1}$

by which the phenomenological coefficients are related to the friction coefficients. The friction coefficients can be determined in the binary subsystems.

\subsection{A solution for short times}

It has been argued that the model by Reuvers et al. is not valid if the concentration at the interface do not remain constant [11]. However, the model is generally valid for short immersion times, also when the interfacial concentrations would change with time.

Immediately after the polymer solution has been brought into contact with the coagulation bath, the concentration profiles are stepwise functions. This causes, mathematically, the fluxes to be infinitely large at $t=0$. In other words, the diffusion behavior features a singular point at $t=0$ and $m=0$. To overcome this, the Boltzmann conversion $[6,7,21,22]$ may be used:

spatial $/$ time coordinate $\zeta=\frac{m}{2 \sqrt{t}}$

time coordinate $\tau=\sqrt{t}$

i.e., the spatial coordinate $m$ is converted to $m$ / $(2 \sqrt{ } t)$ while time is converted to $\sqrt{ } t$. the diffusion equations for the polymer solution then become:

$$
\begin{aligned}
\tau \frac{\partial\left(\frac{\phi_{i}}{\phi_{3}}\right)}{\partial \tau}=\zeta & \frac{\partial\left(\frac{\phi_{i}}{\phi_{3}}\right)}{\partial \zeta} \\
& +\frac{1}{2} \frac{\partial}{\partial \zeta} \sum_{j=1,2,4}\left\{\overline{\mathbf{v}}_{i} \phi_{3} \mathrm{~L}_{i j} \frac{\partial \mu_{j}}{\partial \zeta}\right\}
\end{aligned}
$$

Normally, fluxes in such a problem are dependent on the square root of time. It can be proven [21] that while the time derivatives of the concentrations at $t=0$ and $m=0$ are singular. the $t$ derivatives are not (they are actually constant). For small times therefore: $\left\{\tau \frac{\partial\left(\frac{\phi_{i}}{\phi_{3}}\right)}{\partial \tau}\right\} \rightarrow 0$ when $\tau \rightarrow 0$

One now sees that it is not necessary for the timederivative of the compositions to be zero at the interface, as Reuvers et al. assumed. They may even be infinitely large, as long as the $\tau$ derivative is finite.

It appears that we obtain the same approximation as found by Reuvers et al., but without the assumption of constant interfacial compositions. We have now an approximation valid generally for short times:

$$
\begin{array}{r}
\zeta \frac{\partial\left(\frac{\phi_{i}}{\phi_{3}}\right)}{\partial \zeta}+\frac{1}{2} \frac{\partial}{\partial \zeta} \sum_{j=1,2,4}\left\{\bar{v}_{i} \phi_{3} L_{i j} \frac{\partial \mu_{j}}{\partial \zeta}\right\}=0 \\
i=1,2,4
\end{array}
$$

A similar relation can be found for the coagulation bath.

It is thus shown that for the first moments of immersion the composition path is indeed only dependent on $\zeta$.

\subsection{Calculation procedure}

The true diffusion equations are calculated for a short time (e.g., $0.1 \mathrm{~s}$ ) with the help of the D03PGF-routine from the National Algorithms Group library [23].

The procedure is as follows:

1. An interfacial composition on the binodal is assumed.

2. The diffusion profiles in the polymer solution are calculated.

3. The fluxes to and from the polymer solution are calculated.

4. The fluxes are used to calculate the diffusion profiles in the coagulation bath.

5. The fluxes to and from the coagulation bath are calculated.

6. These fluxes are compared with the fluxes from step 3.

7. The whole procedure is repeated with differ- 
ent interfacial compositions, until the fluxes from step 3 and step 5 are equal.

\subsection{Friction coefficients}

In our model we will use the same assumptions as Reuvers et al. used. That is, quaternary friction coefficients $R_{i j}$ are only dependent on the two components $i$ and $j$. Therefore the binary friction coefficients may be used in the form of:

$R_{i j}^{\text {quaternary }}=R_{i j}^{\text {binary }}\left(\frac{\phi_{i}}{\phi_{i}+\phi_{j}}\right)$

This enables us to use date measured in the limiting binary systems [7].

As a model system, we use the system consisting of: (1) nonsolvent, water; (2) solvent, 1methyl-2-pyrrolidone (NMP); (3) membrane forming polymer, poly (ether sulfone) (PES); and (4) polymeric additive, poly(vinylpyrrolidone) (PVP).

Since on the diffusivity of poly (ether sulfone) in NMP not so many data are available, the relation from Radovanovic et al. [22] for the diffusivity of polysulfone in DMAc is used.

$\frac{v_{3} R T}{M_{2} R_{23}}=18.0 \cdot 10^{-9-4.386 \phi_{3}} \quad\left(\mathrm{~m}^{2} / \mathrm{s}\right)$

DMAc and NMP are substances of similar chemical nature, the experiments that were performed with NMP as solvent could as well have been performed with DMAc as solvent: membrane formation with DMAc gives the same experimental results.

Eq. 9 approximately agrees with the experimental data from Tkacik and Zeman [24,25] on the system PES-NMP-water, and with the values measured in our laboratory. From calculations of the compositions paths it appears that the results are not very sensitive to the exact values of the friction coefficients.

Diffusion coefficients of NMP in water are taken from ref. 25 . The friction coefficient obtained from these data is estimated to be constant:

$\frac{v_{1} R T}{M_{2} R_{12}}=6.0 \cdot 10^{-10} \quad\left(\mathrm{~m}^{2} / \mathrm{s}\right)$
The thermodynamic data from the system PES-NMP-water is based on the work by Tkacik and Zeman [24], and work from our laboratory. The data on the interaction of PVP with other components are given in ref. 26.

\subsection{A system with two polymers in one phase}

In diluted solutions (i.e., below the overlap concentration ), binary diffusion coefficients for low molecular weight substances and polymers are usually inversely related to the square of the molecular weight of the polymer.

Polymer solutions meant for membrane formation are always quite concentrated: a typical concentration in the system considered here is: $25 \mathrm{wt} \%$ membrane-forming polymer and $15 \mathrm{wt} \%$ polymeric additive. A total polymer weight content of $40 \mathrm{wt} \%$ indicates that the polymer solution can be regarded as an intertwined network of polymer molecules 'swollen' with solvent and nonsolvent molecules. Since the two polymers that are used mix well, they will be well entangled.

De Gennes [27] has developed theoretical ideas on such concentrated solutions. According to his ideas, such a solution can be regarded as consisting of 'blobs', parts of a polymer chain that mainly interact with themselves, and that do not interact with other chains. The number of blobs is related to the concentration of the polymers. In rather concentrated solutions, the properties of the solution are determined by the properties of the blobs, and not by the properties of the complete chains. This situation is schematically shown in Fig. 2a. The molecular weight of the polymers has no effect on diffusion of solvent (or nonsolvent) molecules through such a network of blobs, since the molecular weight of a blob is much smaller than that of the complete polymer chain. Therefore, above the overlap concentration, the diffusion coefficient between a solvent and a polymer is independent of the molecular weight of the polymer.

Diffusion of one of the polymer molecules through the solution, however, is quite slow. A polymer chain can only move along its long axis. One may visualize this as movement of the poly- 


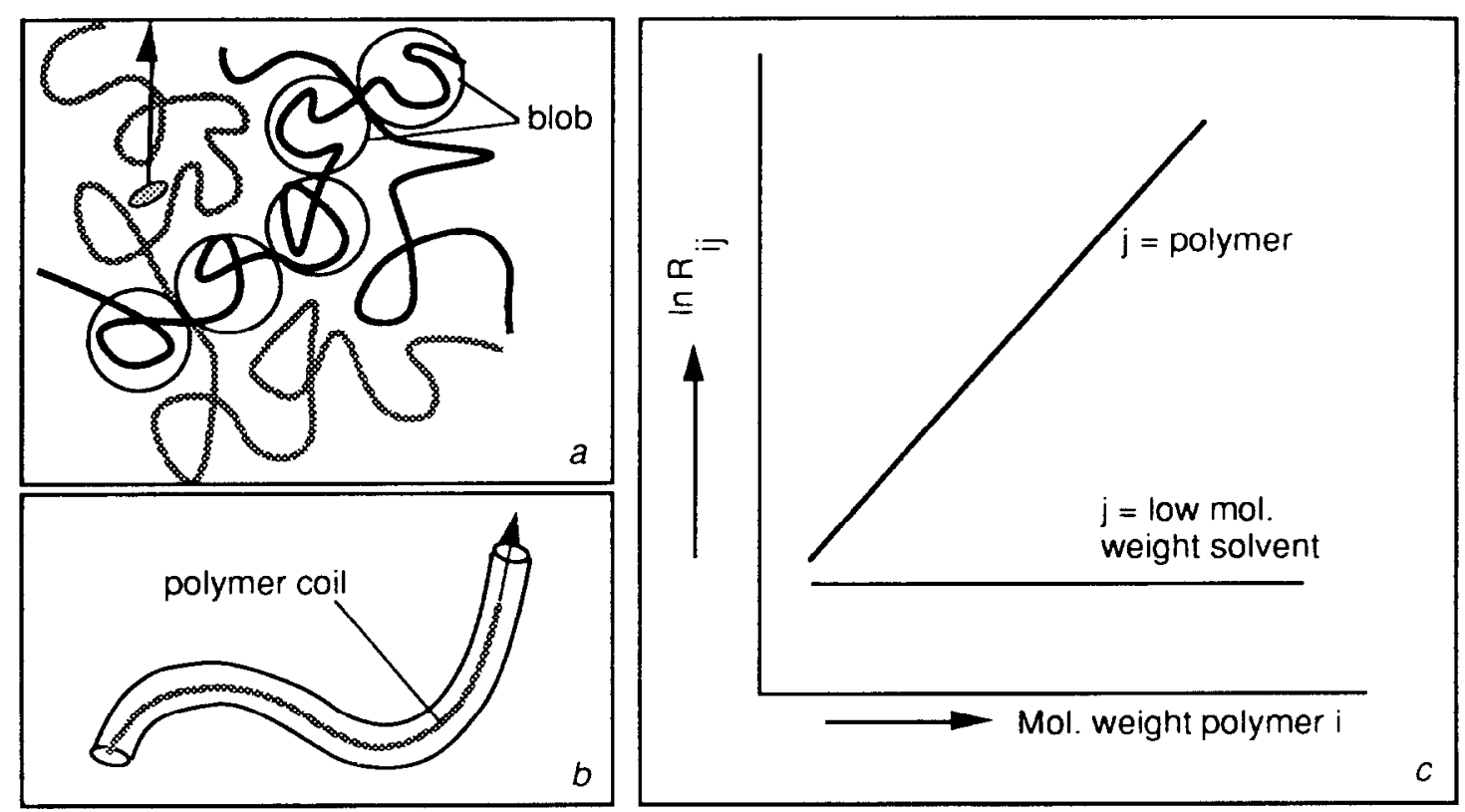

Fig. 2. A schematic picture of diffusion processes in a concentrated solution. Nonsolvent (1) and solvent (2) can diffuse through the network of blobs (a), while movement of polymeric molecules with respect to other polymer molecules is restricted to movement through a 'tube' (reptation; b). The consequence is shown in (c): the friction coefficient between two polymers $(i$ and $j$ ) increases with the square of the molecular weight; the friction coefficient between a low molecular weight component $(j)$ and a polymer $(i)$ is independent on the molecular weight of the polymer.

mer coil in a tube, shown in Fig. 2b; while the polymer coil is moving, new parts of the tube are created at one end, while at the other end parts of the tube are destroyed [27]. De Gennes showed that in this situation the diffusion coefficient is inversely proportional to the square of the molecular weight of the polymer(s).

Summarizing, for concentrated solutions (i.e., clearly above the overlap concentration), the diffusion rate between solvent and polymer is independent of the molecular weight of the polymer; the polymer-polymer diffusion rate is quite dependent on the molecular weight. This is schematically shown in Fig. $2 c$.

The implication of this is, that accordingly the mass exchange of solvent and nonsolvent is much faster than the diffusional processes between the polymers. During the very first moments of immersion, the polymeric additive does not have the possibility to move at all relative to the membrane-forming polymer. The two polymers behave as one polymeric network.
In our mass transfer model, which is only meant for the first moments of immersion, one can conclude that the friction coefficient between the two polymers is very large when compared to the other friction coefficients in the system. We can therefore assume that the velocity difference between the two polymeric components is negligible compared to the other velocity differences in the diffusion system.

The condition of no movement between the two polymers imposes two alterations on the system.

The first one concerns the mass transfer model. Since the polymers behave as one single polymer, the system reduces into a semi-ternary system. The phenomenological coefficients can be calculated from.

$\mathbf{L}=\left[\begin{array}{c}-\frac{1}{c_{1}}\left(c_{2} R_{12}+c_{3} R_{13}+c_{4} R_{14}\right) \\ R_{12}\end{array}\right.$ 


$$
\left.-\frac{1}{c_{2}}\left(c_{1} R_{12}+R_{12} R_{23}+c_{4} R_{24}\right)\right]^{-1}
$$

Comparison with the analogous expressions by Reuvers et al. shows that the polymer network behaves as a weighted average over the two polymeric components.

The second adaptation to be made is thermodynamic in nature. During the first moments of immersion, the assumption of local equilibrium at the interfacial boundary cannot be maintained.

During phase separation, the two polymers present always tend to separate, regardless ot their mutual miscibility, as long as one polymer is mainly soluble in only one phase (the membrane-forming polymer) and the other polymer is soluble in both phases (the polymeric additive). This has entropic reasons: two polymers have very low entropy of mixing. This effect has been clearly shown earlier [26].

For the initial stage we can only assume local equilibrium for the low molecular weight components, solvent and nonsolvent, since they can move freely through the polymeric network and the coagulation bath. We pose on the system the restriction that all polymer remains present in the polymer phase, and that in the other phase no polymer is present at all. In the next paragraph this restriction will be worked out in detail.

\subsection{Thermodynamics for the first moments of immersion}

As was discussed before, the phase behavior at the interface for short times is different from the equilibrium phase behavior for the quaternary system. Due to the kinetic restrictions of the system, we have to assume that the polymers act as one single polymeric network.

The phase diagram for the first moments was calculated by assuming equilibrium for the solvent and nonsolvent, while forcing the polymer concentrations in the diluted phase to be zero. The polymer concentrations in the concentrated phase were chosen as independent variables.

For the concentrated phase, the following re- lations were used to describe the chemical potentials of solvent and nonsolvent [26]:

$$
\begin{aligned}
& \frac{\Delta \mu_{1}}{R T}=\ln \phi_{1}-s \phi_{2}-r \phi_{3}-t \phi_{4}+\left(1+g_{12} \phi_{2}\right. \\
& \left.+\chi_{13} \phi_{3}+g_{14} \phi_{4}\right)\left(1-\phi_{1}\right)-s g_{23} \phi_{2} \phi_{3} \\
& -r g_{34} \phi_{3} \phi_{4}-s g_{24} \phi_{2} \phi_{4}-\phi_{2} u_{2}\left(1-u_{2}\right) \\
& \quad \times\left(\frac{\partial g_{12}}{\partial u_{2}}\right)-\phi_{4} u_{4}\left(1-u_{4}\right)\left(\frac{\partial g_{14}}{\partial u_{4}}\right) \\
& \frac{s \Delta \mu_{2}}{R T}=s \ln \phi_{2}-\phi_{1}-r \phi_{3}-t \phi_{4}+\left(s+g_{12} \phi_{1}\right. \\
& \left.\quad+s g_{23} \phi_{3}+s g_{24} \phi_{4}\right)\left(1-\phi_{2}\right) \\
& \quad-\chi_{13} \phi_{1} \phi_{3}-g_{14} \phi_{1} \phi_{4}-r g_{34} \phi_{3} \phi_{4} \\
& \quad+\phi_{1} u_{2}\left(1-u_{2}\right)\left(\frac{\partial g_{12}}{\partial u_{2}}\right)-s \phi_{3} v_{2}\left(1-v_{2}\right) \\
& \quad \times\left(\frac{\partial g_{23}}{\partial v_{2}}\right)-s \phi_{4} w_{2}\left(1-w_{2}\right)\left(\frac{\partial g_{24}}{\partial w_{2}}\right)
\end{aligned}
$$

while for the diluted phase, binary equations were used (no polymer is present here).

$$
\frac{\Delta \mu_{1}}{R T}=\ln \phi_{1}-s \phi_{2}+\left(1+g_{12} \phi_{2}\right) \phi_{2}-\phi_{2}^{2} \phi_{1}\left(\frac{\partial g_{12}}{\partial \phi_{2}}\right)
$$

$$
\begin{gathered}
\frac{s \Delta \mu_{2}}{R T}=s \ln \phi_{2}-\phi_{1}+\left(s+g_{12} \phi_{1}\right) \phi_{1}+ \\
\phi_{1}^{2} \phi_{2}\left(\frac{\partial g_{12}}{\partial \phi_{2}}\right)
\end{gathered}
$$

In these relations, the binary interaction parameters were assumed to be only dependent on the two components indicated by the interaction parameter itself. The following parameters were introduced [28]:

$g_{12}$ is dependent on $u_{2}=\phi_{2} /\left(\phi_{2}+\phi_{1}\right)$

$g_{23}$ is dependent on $v_{2}=\phi_{2} /\left(\phi_{2}+\phi_{3}\right)$

$g_{14}$ is dependent on $u_{4}=\phi_{4} /\left(\phi_{4}+\phi_{1}\right)$

$g_{24}$ is dependent on $w_{2}=\phi_{2} /\left(\phi_{2}+\phi_{4}\right)$ 
$g_{34}$ is dependent on $v_{3}=\phi_{3} /\left(\phi_{3}+\phi_{4}\right)$

Any concentration dependence of $\chi_{13}$ cannot be measured (since components 1 and 3 are not miscible); the absence of concentration dependence is assumed [4]. Therefore, the symbol $\chi$ was used instead of $g$. The entropic parameters are the ratios of the molar volumes of the different species:

$s=\frac{\bar{v}_{1} M_{1}}{\bar{v}_{2} M_{2}} ; \quad r=\frac{\bar{v}_{1} M_{1}}{\bar{v}_{3} M_{3}} ; \quad t=\frac{\bar{v}_{1} M_{1}}{\bar{v}_{4} M_{4}}$

in which $\bar{v}_{i}$ is the specific volume and $M_{i}$ is the molecular weight of component $i$.

For the calculation, the usual numerical procedure is used (see Hsu and Prauznitz [3], and Altena and Smolders [4]). The chemical potentials as defined by Eqs. (12) to (15) were set equal for each component by varying the nonsolvent concentrations in both phases. The concentrations of components 3 and 4 were fixed in one phase, component 2 was determined by the mass balance.

\section{Experimental}

PES, Victrex 5200 P, supplied by ICI Ltd., was dried at least for $12 \mathrm{~h}$ at $80^{\circ} \mathrm{C}$ before usage. No further purification was applied. PVP, grade K30 and K90 from Jansen Chimica, was used as received. In Table 2 the molecular weights of the various polymers are given.

NMP was obtained from Merck, synthesis grade, and was used without further purification. Water was demineralized and ultrafiltered. The polymer solutions were cast with a thickness of $0.2 \mathrm{~mm}$ on a glass plate.

The setup used for the transmission experi-

Table 2

Molecular weights of the polymers used, measured by gel permeation chromatography

\begin{tabular}{lrc}
\hline Polymer & $M_{\mathrm{n}}(\mathrm{g} / \mathrm{mol})$ & $M_{\mathrm{w}}(\mathrm{g} / \mathrm{mol})$ \\
\hline PES Victrex 5200 P & 22,300 & 43,800 \\
PVP K30 & 8,700 & 18,100 \\
PVP K90 & 99,800 & 228,200 \\
\hline
\end{tabular}

ments [9] is shown in Fig. 3. Tables 3 and 4 show all polymer solutions and coagulation baths used. All transmission profiles were measured in triplo.

\section{Results}

\subsection{Calculations on thermodynamics}

Phase diagrams calculated in this way are shown in Fig. 4a to $h$. In these figures the thermodynamic parameters are varied. Since these binodals are only valid for very short times, and are completely different from the real binodal, one might call these curves virtual binodals.

It appears from Fig. 4a that the addition of component 4 (PVP) decreases the size of the demixing gap: the solution becomes more compatible with the nonsolvent It should be remembered that the real cloudpoint curves of these systems (i.e., when kinetics are not hampered) are $\sim 1$ vol\% of nonsolvent or less [26]; the solutions are in reality very incompatible with the nonsolvent. Nevertheless, for short times, Fig. $4 a$ shows that the solution can contain up to 50 vol\% of nonsolvent (this is as long as no movement between the two polymers is possible). This is not very dependent on the molecular weight of the additive, as long as the additive is still macromolecular in nature. This is shown in Fig. $4 \mathrm{~b}$. When the molecular weight of the additive becomes too low (i.e., $<5000 \mathrm{~g} / \mathrm{mol}$ ), the binodal shifts even further to the right. Since we already need a high molecular weight for the additive to hinder the diffusion between the two polymers (e.g., several tens of thousands $\mathrm{g} / \mathrm{mol}$ ), we can assume that the actual molecular weight has no influence on these virtual binodals.

Figs. $4 \mathrm{c}$ and $4 \mathrm{~d}$ show the effects of the parameters that are already present in a ternary system without component 4 (PVP); we see the same behavior as in a ternary system [4]. It is observed that polymer-solvent interactions, $g_{23}$ and $g_{24}$, are not important, of course as long as miscibilities are ensured (see Figs. $4 \mathrm{e}$ and $4 \mathrm{f}$ ). The interaction between the polymeric additive and the nonsolvent, $g_{14}$ (Fig. $4 \mathrm{~g}$ ) is analogous to $g_{13}$.

The interaction between the two polymers, $g_{34}$, 


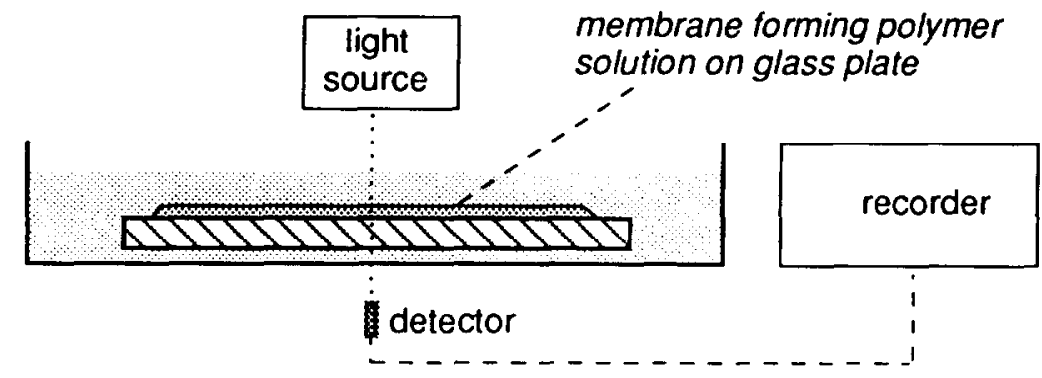

Fig. 3. Experimental setup to measure light transmission through the polymeric solution during the immersion precipitation step [9].

Table 3

Polymer solutions

\begin{tabular}{rlrl}
\hline No. & wt\% PES & wt\% PVP & PVP type \\
\hline 1 & 15 & 5 & K30 \\
2 & 15 & 10 & K30 \\
3 & 15 & 15 & K30 \\
4 & 15 & 20 & K30 \\
5 & 15 & 5 & K90 \\
6 & 15 & 10 & K90 \\
7 & 15 & 15 & K90 \\
9 & 20 & 5 & K30 \\
10 & 20 & 10 & K30 \\
11 & 20 & 15 & K90 \\
12 & 20 & 5 & - \\
13 & 20 & 10 & - \\
14 & 15 & 0 & \\
15 & 20 & 0 & \\
\hline
\end{tabular}

Table 4

Coagulation baths

\begin{tabular}{ll}
\hline Bath No. & wt\% NMP \\
\hline 1 & 0 \\
2 & 10 \\
3 & 20 \\
4 & 30 \\
5 & 40 \\
6 & 50 \\
7 & 60 \\
8 & 70 \\
9 & 75 \\
10 & 80 \\
\hline
\end{tabular}

seems not to have any influence (again as long the polymers are still miscible), as shown in Fig. $4 \mathrm{~h}$. Soon after $g_{34}$ becomes positive, the poly- mers become immiscible, and the situation becomes more complicated. This region is, however, outside the scope of this paper, which focuscs on two-phase equilibria.

Generally, from Fig. 4 it may be concluded that for the short-term thermodynamic behavior of the polymer solution only the interactions with the nonsolvent are important, i.e., the parameters $g_{12}, \chi_{13}$ and $g_{14}$ (see Figs. $4 \mathrm{c}, 4 \mathrm{~d}$, and $4 \mathrm{~g}$ ). The influence of the solvent-nonsolvent interaction parameter has been well investigated by Smolders and coworkers $[4,8]$. We may say that in our quaternary system, this influence looks analogous. The same can be said of the polymernonsolvent interaction parameter. The influence of the additive seems to be mainly governed by the interaction between the additive and the nonsolvent, $g_{14}$. This can be easily understood. If the value of this parameter were the same as the value of $\chi_{13}$, there should be no shift of the binodal whatsoever, the additive should behave exactly the same as the membrane-forming polymer. The parameter $g_{14}$ is therefore a measure of the difference between the two polymers.

To summarize the effects shown in Fig. 4, it appears that the thermodynamics during the first moments of immersion are mainly governed by one parameter, the nonsolvent-additive parameter, which expresses the "hydrophilicity" (when the nonsolvent is water) of the additive. Apart from effects from the basic ternary membraneforming system without polymeric additive (effects of $g_{12}, g_{23}$, and $\chi_{13}$ ), the system is relatively insensitive to the other parameters in the system. 

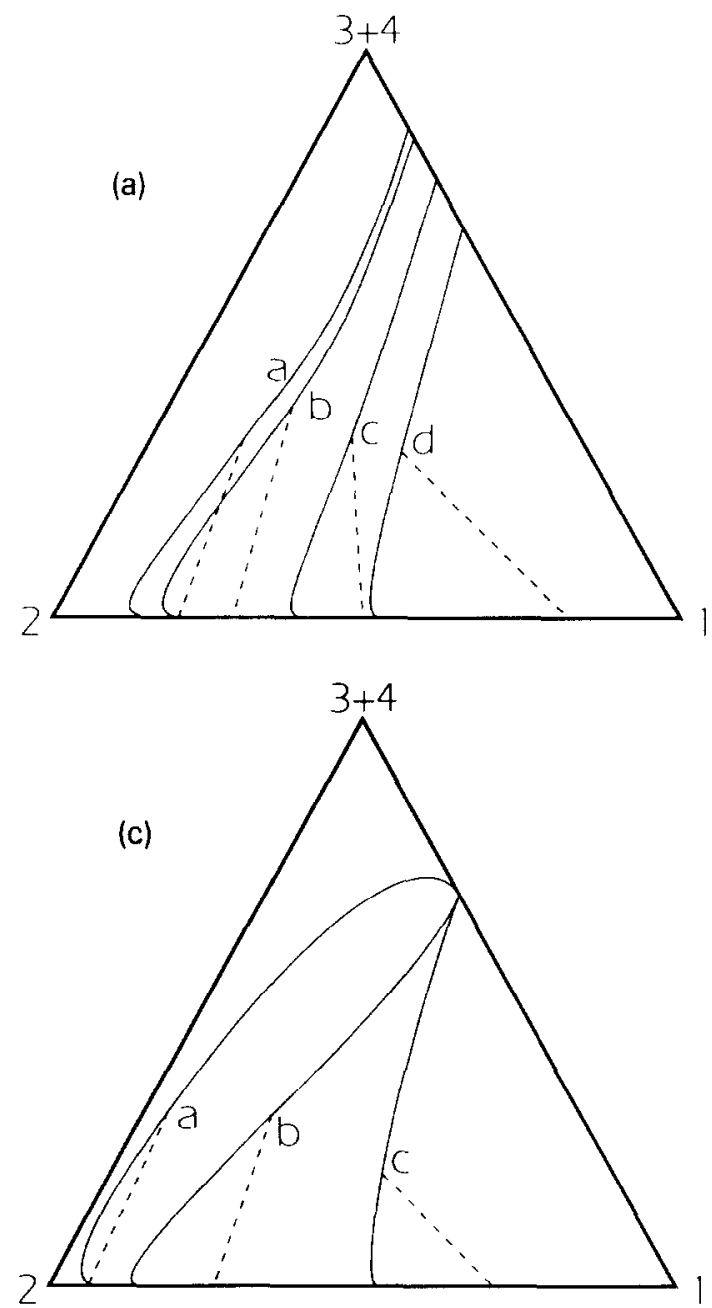

\subsection{Calculations on mass transfer}

The "short-term" thermodynamics (valid for the first seconds of immersion) as shown above was used as the basis for the calculation of the composition paths for the system.

Fig. 5 shows a typical initial composition path. The nonsolvent flux through the interface is much higher than in the ternary system, and that it is comparable to the solvent flux.

Preliminary measurements performed in our laboratory indicated that the diffusivity of PVP is of the same magnitude as the diffusivity of PES. We therefore assumed that they were the
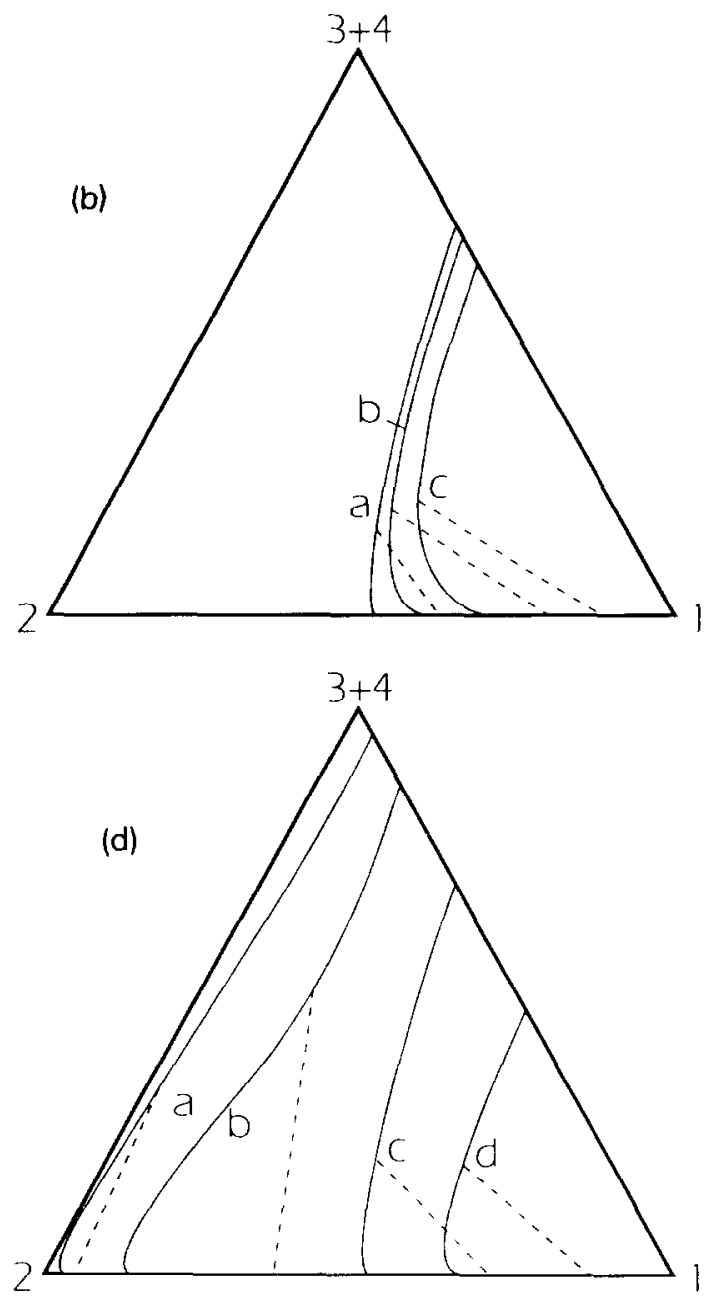

same and possessed the same concentration dependency.

Fig. 6 shows the influence of a deviation from this assumption. It appears that although there are deviations, the composition path remains largely the same when the diffusivities are changed over two orders of magnitude. Apparently, the composition paths are not very sensitive to exact values of the friction coefficients. Since we have only few exact data on the system, this is an important conclusion.

On the basis of the preceding figures, we now may look into the behavior of the systems more closely. In Fig. 7 the ratio of additive to polymer in the system is varied from zero to unity. A 

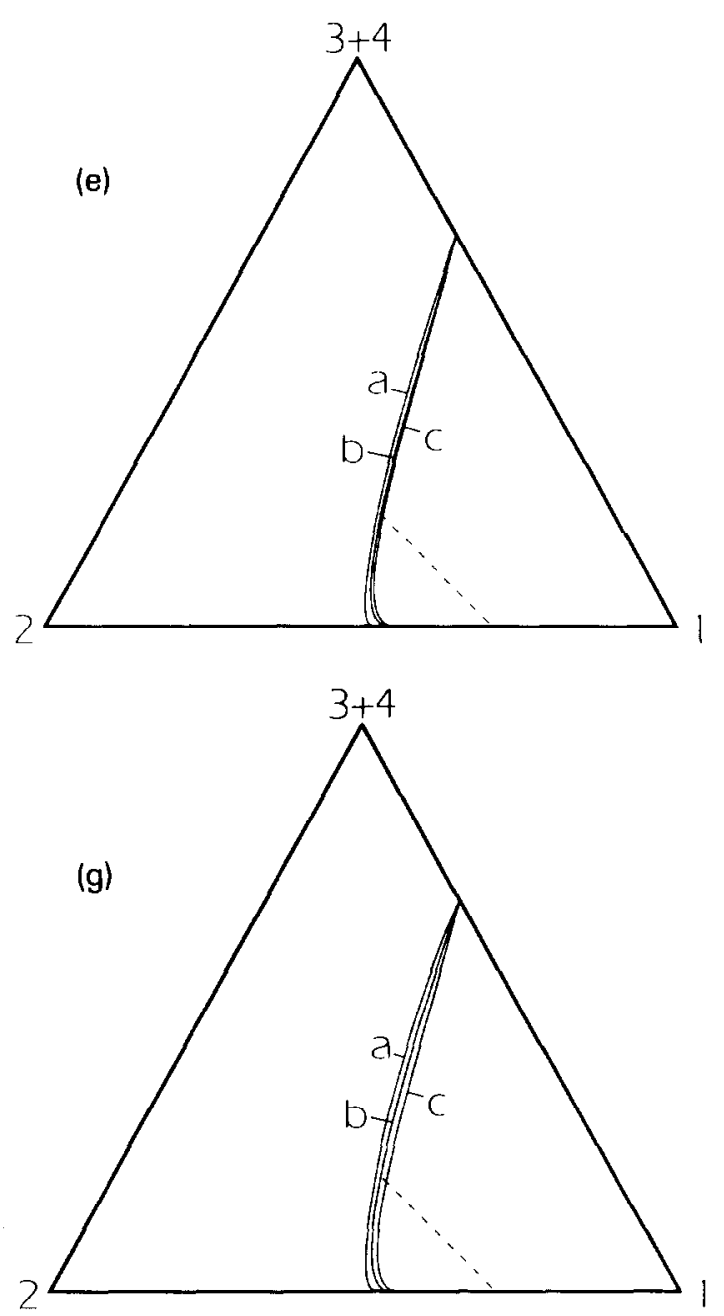


Fig. 4. Phase diagrams for the first moments of immersion. The following parameters were used unless indicated otherwise: $g_{12}=1.0, \chi_{13}=1.5, g_{14}=0.5, g_{24}=0.5, g_{23}=0.5, g_{34}=-1 ; s=0.182, r=0.001 ; t=0.001 ; \phi_{3} / \phi_{4}=1$. In each diagram one variable is varied. Dotted lines are tielines; uninterrupted lines are virtual binodals. (a) Variation of the ratio of additive to polymer between 0 and 1. (b) Variation of the molecular weight of the additive between 500 and $500,000 \mathrm{~g} / \mathrm{mol}$ (c) variation of $g_{12}$ between 0.0 and 1.0. (d) Variation of $\chi_{13}$ between 4.0 and 1.0. (e) Variation of $g_{23}$ between -1.0 and 0.5 . (f) Variation of $g_{24}$ between -1 and 0.5 . (g) Variation of $g_{14}$ between 1.0 and 0.0 . (h) Variation of $g_{34}$ between -1 and 0.

characteristic feature appears with increasing $\phi_{4} /$ $\phi_{3}$, viz. a lowering in polymer concentration in the top layer. When the ratio $\phi_{4} / \phi_{3}$ is enlarged further, the interfacial polymer concentration stays approximately constant. From the calculations it appears that nonsolvent fluxes through the interface increase approximately three-fold when increasing the ratio from zero to 0.25 ; after this they remain approximately the same.

We can see that the initial composition path without any addition, which is typically of an instantaneous demixing type, shifts to a delay of demixing type when additive is present. No demixing takes place as long as the two polymers cannot move relative to each other. Of course we should remember that this assumption holds only for the first few moments. After this initial stage the two polymers start to move relative to each other. The demixing itself is determined by the demixing between the two polymers [26]. Re- 


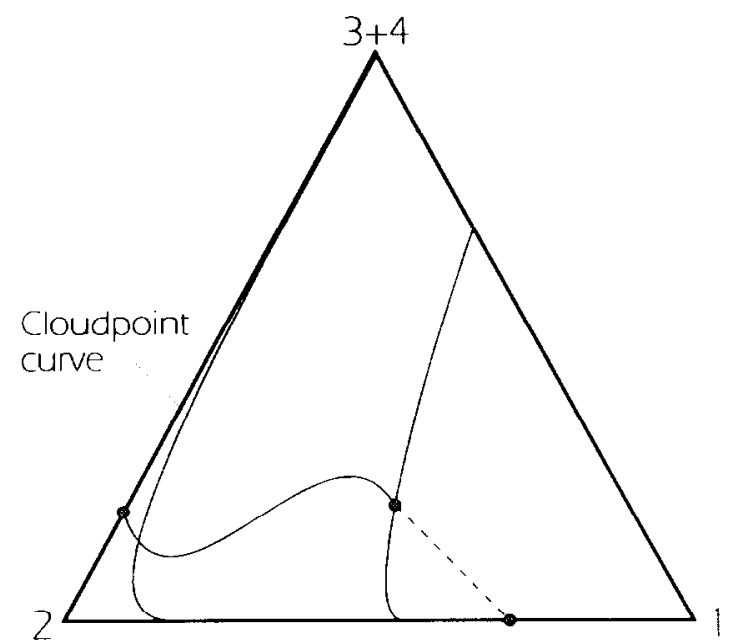

Fig. 5. An initial composition path for a system with an equal amount of additive 4 and polymer 3 . The friction coefficients $R_{i 4}$ are chosen as equal to the friction coefficients $R_{i 3}$. Other parameters are as in Fig. 4, and as indicated in the text. Initial composition of the polymer solution: $20 \mathrm{vol} \%$ polymer $(3+4$, PES + PVP $)$ in solvent. Initial composition of the coagulation bath: pure nonsolvent.

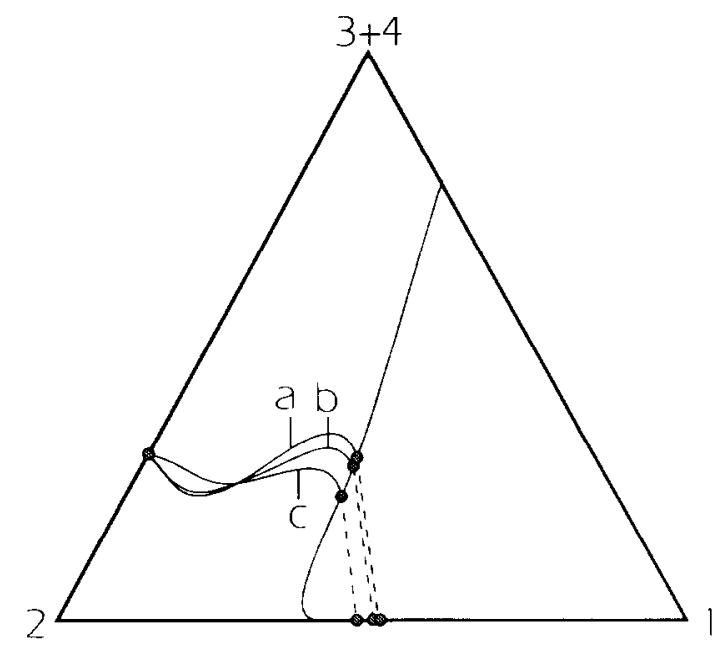

Fig. 6. Initial composition paths that show the influence of the choice of values for $R_{i 4}$ : (a) $R_{i 4}=0.1 R_{i 3}$; (b) $R_{i 4}=R_{i 3}$ and (c) $R_{i 4}=10 R_{i 3}$. Other parameters and concentrations are as in Fig. 5.

garding the mechanism of formation of macrovoids, the behavior of the solution immersed in a coagulation bath that contains increasing amounts of solvent is of importance.

In the basic ternary membrane-forming sys-

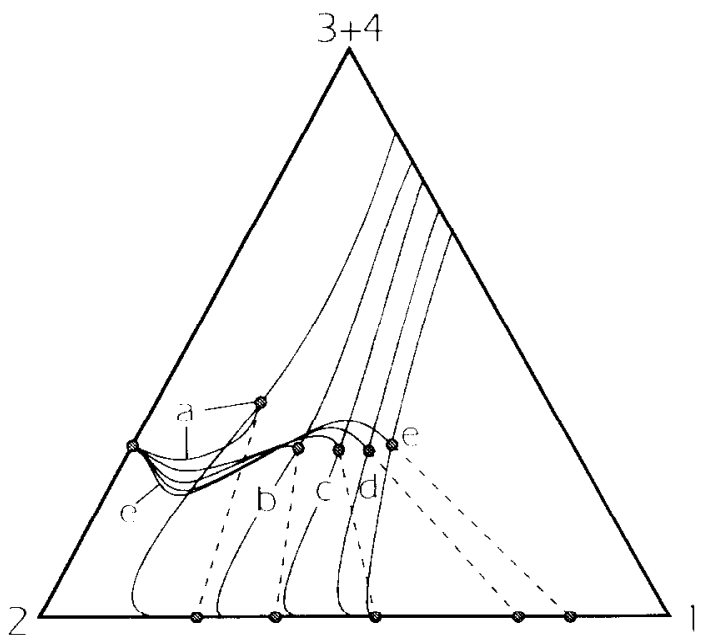

Fig. 7. Initial composition paths with a varying amount of additive in the polymer solution. Ratios of additive (4) to polymer (3) are (a) 0 , (b) 0.25 , (c) 0.5 , (d) 0.75 , (e) 1.0 . Other parameters and concentrations are as in Fig. 5. Dashed lines represent the (local) equilibrium at the interface.

tem, an increase of the solvent concentration in the coagulation bath results in a slowing down of the diffusion of nonsolvent. Above a certain solvent concentration, the instantaneous type of demixing is replaced by a delayed type of demixing.

Fig. 8 shows the influence of the composition of the coagulation bath for a quaternary system, from zero to $60 \mathrm{vol} \%$ of solvent. As we can see, the polymer concentration at the interface decreases to zero: at around $50 \mathrm{vol} \%$ of solvent in the coagulation bath, there is no discrete interface to prevent membrane-forming polymer to diffuse into the coagulation bath. Since our model is based on the stagnancy of components 3 and 4 (PES and PVP), and is developed only for short times, these initial composition paths should not be taken too literally, although they might still be used qualitatively. What one expects on the basis of Fig. 8 is the following. When the solvent concentration in the coagulation bath increases, the polymer concentration in the top layer drops to zero. Initially this means that the membraneforming polymer (together with the additive) dissolves in the coagulation bath.

In the polymer solution near the interface, the polymer concentration becomes very low, allow- 


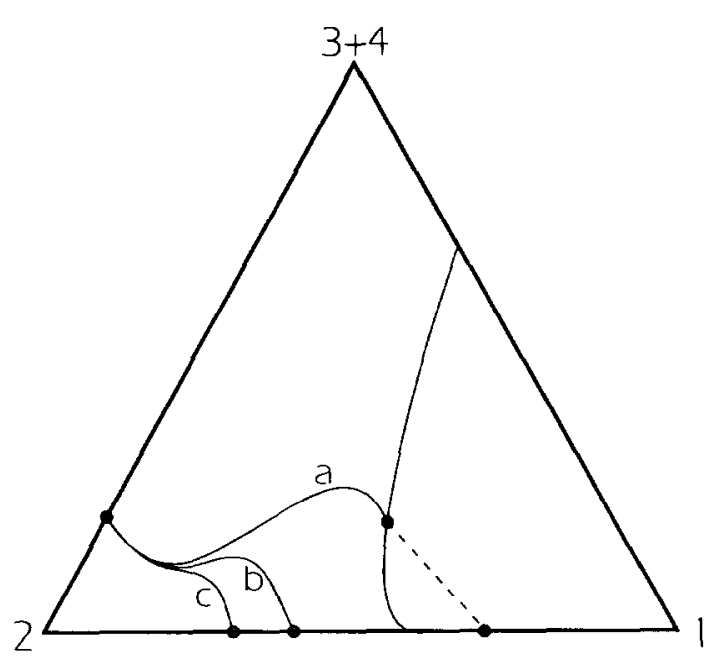

Fig. 8. Initial composition paths with a varying coagulation bath: (a) pure nonsolvent, (b) 40 vol\% solvent, (c) 60 vol\% solvent. All concentrations and parameters are as in Fig. 5.

ing relatively fast polymer-polymer interdiffusion. When this interdiffusion has become possible, the compositions in the top layer are very instable, they are actually situated inside the spinodal area of the normal phase diagram. This solution will therefore relatively quickly demix according to a spinodal decomposition mechanism. It is important to notice that under conditions that would normally cause delay of demixing (i.e., high solvent concentration in the coagulation bath ), the use of a polymeric additive which is soluble in the nonsolvent makes delay of demixing impossible as a membrane forming mechanism.

\subsection{Transmission measurements}

Fig. 9 shows light transmittance measured as a function of time from the start of membrane formation as recorded for the ternary system (no PVP added). One can see that there is a clear transition from instantaneous demixing to delay of demixing.

This is different when PVP is added to the polymer solution. In Fig. 10 the transmittance profiles are given for different polymer solutions, under variable coagulation conditions.

From 0 to $60 \mathrm{wt} \% \mathrm{NMP}$ in the coagulation

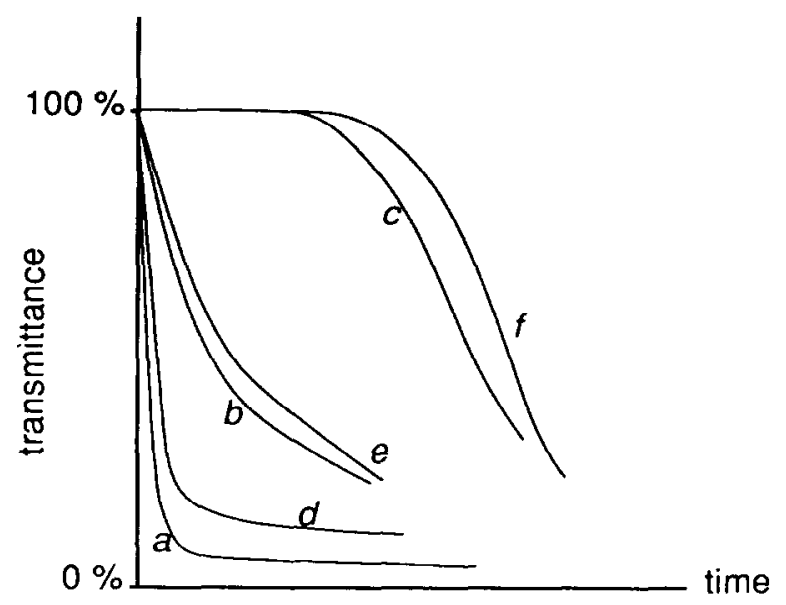

Fig. 9. Transmission profiles for membranes with (a-c) 15 and (d-f) 20 wt\% PES in NMP, and varying amounts of NMP in the coagulation bath: (a,d) $0,(b, d) 60,(c, f) 75$ wt\%. No PVP was used.

bath, roughly the same transmittance profiles are obtained, although the absolute velocity of decrease of the transmittance is higher with less solvent in the coagulation bath. The differences between the different polymer solutions remain the same. Therefore, only the profiles for $60 \mathrm{wt} \%$ NMP and higher in the coagulation bath are shown.

Up to $60 \mathrm{wt} \%$ NMP in the coagulation bath, all profiles indicate instantaneous demixing. It appears that addition of PVP slows down the demixing processes.

At $70 \mathrm{wt} \%$ NMP in the coagulation bath, membranes without PVP show delay of demixing. Addition of a moderate amount of PVP ( $>5$ $w t \%)$ to the polymer solution induces instantaneous demixing. A large amount of PVP K30 added ( $\geq 15 \mathrm{wt} \%$ ) results in a minimum in the transmittance, after which the transmittance temporarily increases again. The more PVP is added, the stronger this effect. The minimum cannot be observed at 70 wt\% NMP with PVP $\mathrm{K} 90$, although irregularities in the profiles could indicate that the effect is present.

Although the initial delay of demixing is suppressed (the transmittance starts to decrease earlier), later on the transmittance is decreasing more slowly than without the use of PVP. Sum- 


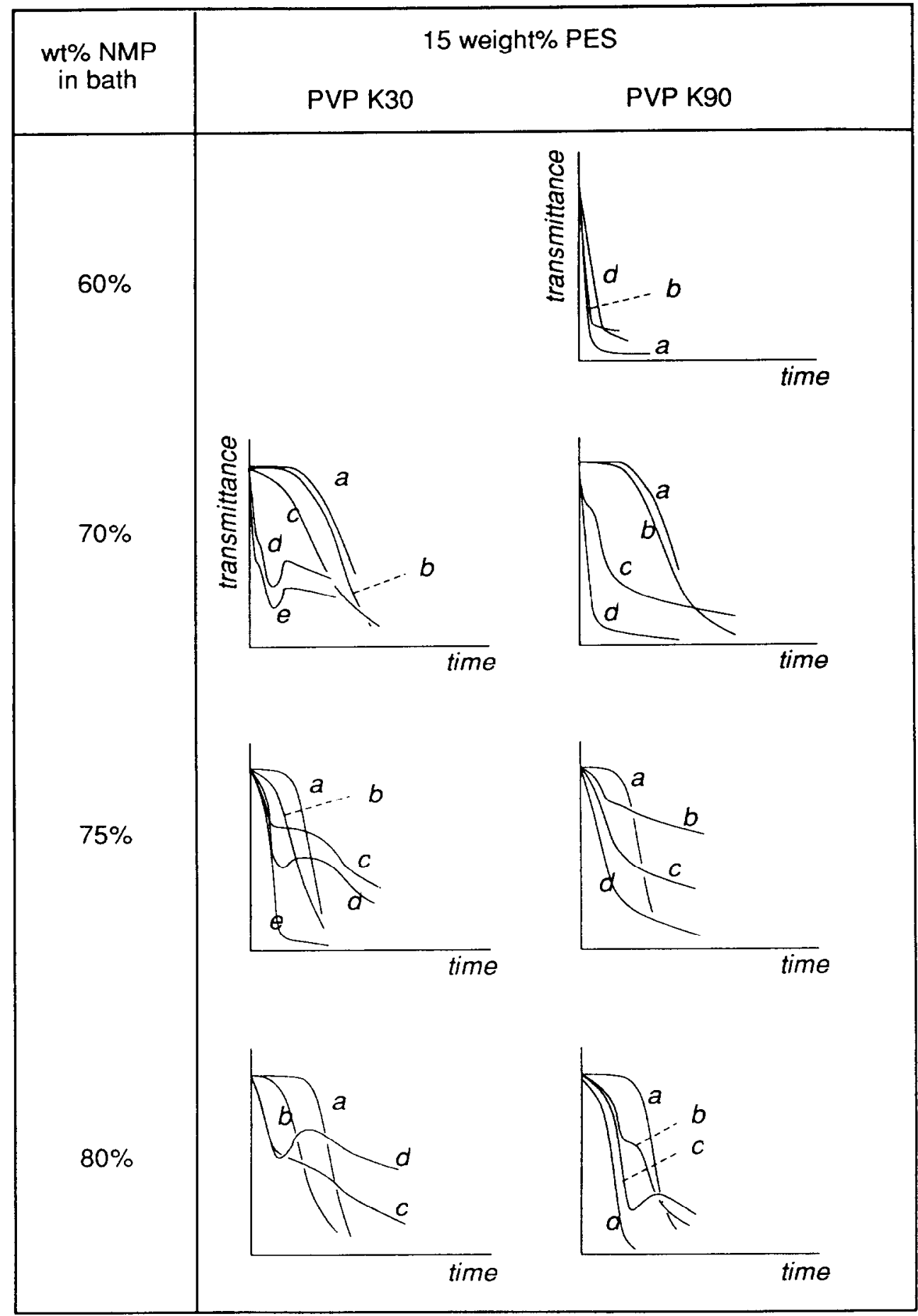




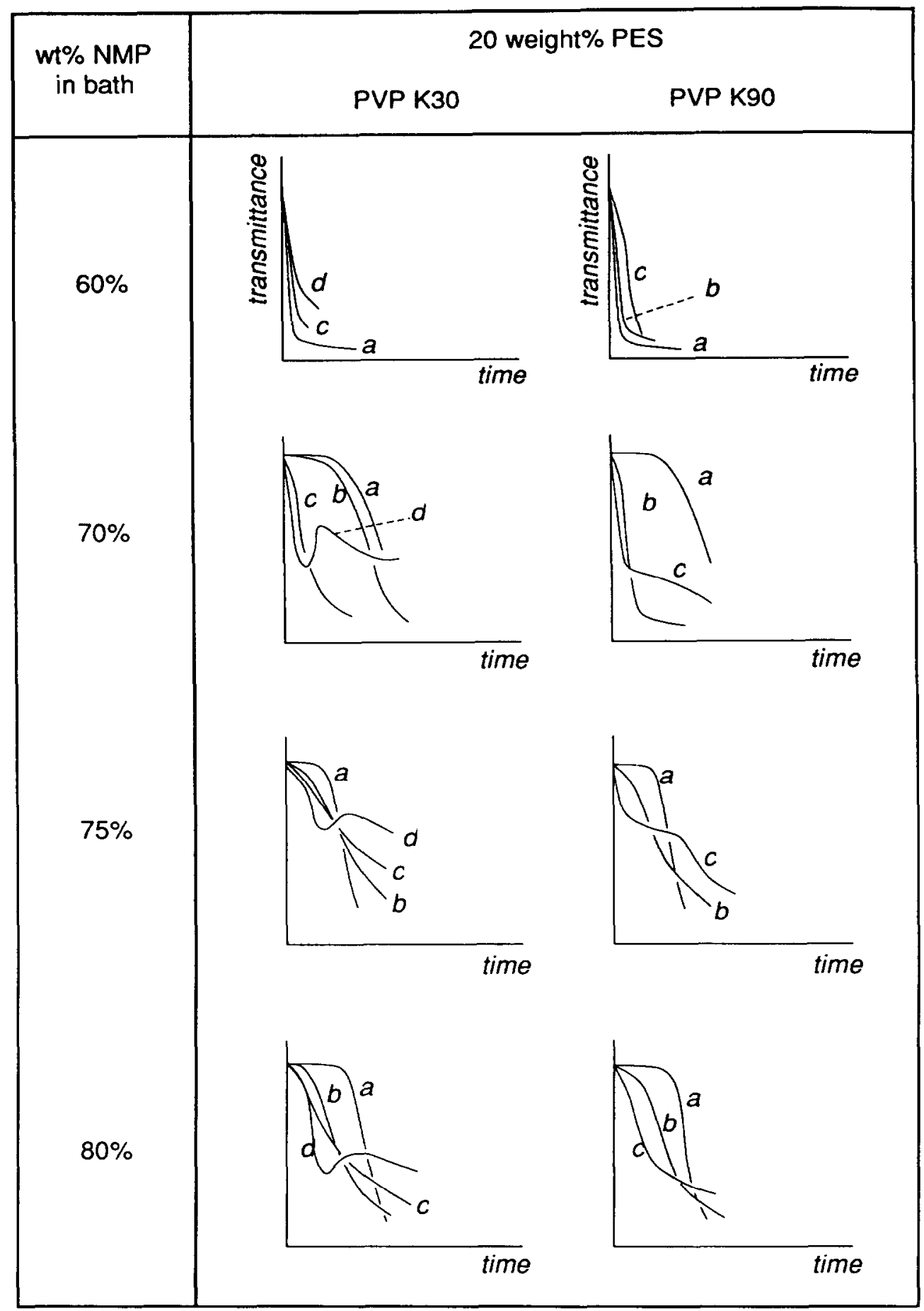

Fig. 10. Recorded transmittance profiles. Indices reflect the PVP concentrations of the polymer solutions: (a) 0, (b) 5, (c) 10, (d) 15, (e) 20 wt\% PVP. 
marizing: demixing starts earlier, but proceeds more slowly by addition of PVP.

A coagulation bath of $75 \mathrm{wt} \% \mathrm{NMP}$ in water also shows the minimum in transmittance. The slowing down of the decrease in transmittance by PVP is more pronounced here. The initial rate of decrease in transmittance is quite large when PVP is used; the demixing process is clearly instantaneous.

At $80 \mathrm{wt} \%$ of NMP in the coagulation bath, one can also see the minimum in transmittance with the solutions of $15 \mathrm{wt} \%$ PES and PVP K90.

Addition of more solvent to the coagulation bath lowers the velocity of demixing, as is expected.

Delay of demixing can only be somewhat preserved when small concentrations of low molecular weight PVP are used.

From the proposed mechanism, it can be seen that experimentally no delay of demixing should be observed when a significant amount of PVP is used. The cloudpoint curve for the systems considered is situated at a few percent of nonsolvent (water) [7], see Fig. 5. When the water concentration in the polymer solution becomes higher, the two polymers in the system will phase separate. During the first moments of immersion, compositions are created that contain much more water than a few percent [7], as shown in Fig. 5. This is even true when, e.g., 70 or $80 \mathrm{vol} \%$ of solvent in the coagulation bath is used. Although delay of demixing is obtained according to the thermodynamic regime valid for the first moments of immersion, the compositions created in the polymer solution are so rich in water that the polymers phase separate. Even for very high concentrations of solvent in the coagulation bath, demixing takes place relatively quickly. Therefore, delay of demixing is suppressed by phase separation between the two polymers. This is confirmed by the transmission profiles shown in Fig. 10. Addition of PVP inhibits the occurrence of delay of demixing up to very high concentrations of solvent in the coagulation bath.

Only at very high solvent concentrations (e.g., $85 \mathrm{wt} \%$ ) no instantaneous demixing is obtained. The term 'delay of demixing' is not appropriate here, since the polymer solution simply dissolves

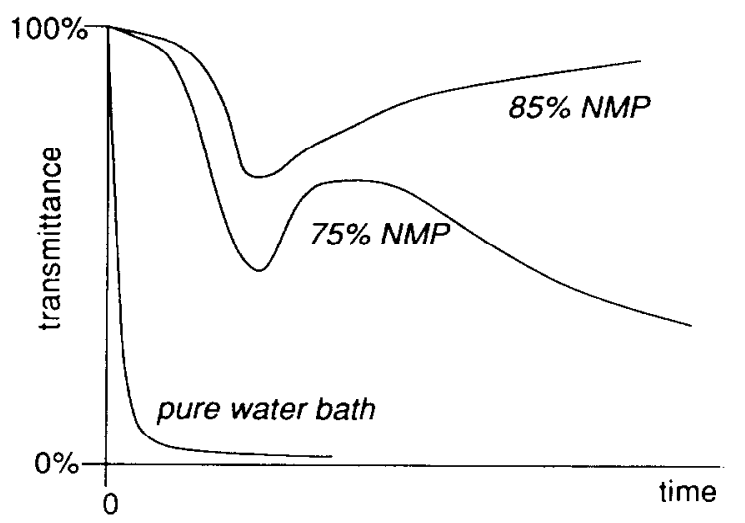

Fig. 11. Transmittance profiles for a solution of $15 \mathrm{wt} \%$ PES and $20 \mathrm{wt} \%$ PVP, with 0,75 and $85 \mathrm{wt} \%$ NMP in the coagulation bath. Other polymer solutions showed similar behavior. The coagulation bath with $85 \mathrm{wt} \%$ NMP did not yield a membrane after an initial decrease in transmission, all material dissolved in the coagulation bath.

in the coagulation bath. Still, instead of giving no demixing at all, the films first become slightly turbid (indicating some phase separation between the two polymers), before they dissolve into the coagulation bath. Even now, delay of demixing is apparently not taking place. Fig. 11 shows this behavior and how it compares with the behavior at lower solvent concentration in the coagulation bath. It appears therefore that the theoretical expectations are confirmed.

\section{Conclusions}

A mass transfer model for a quaternary membrane-forming system is developed for the first moments of immersion.

It is shown that a particular system, incorporating two polymers in the same solution, has some specific characteristics. By using an additive with a certain minimum molecular weight the movement between the two polymers is negligible initially, compared to the movements of nonsolvent and solvent. It is therefore possible to distinguish two different time scales during membrane formation with such a system.

(1) During the shorter time scale, movement of one polymer compared to the other can be assumed zero. Only solvent and nonsolvent are ex- 
changed. A high nonsolvent concentration in the polymer solution results.

(2) In the longer time scale, the movement between the two polymers is possible again. The polymeric additive forms a second (pore) phase; the membrane-forming polymer (4) forms the membrane.

It is essential that the molecular weight of the polymeric additive is high enough to decrease the polymer-polymer diffusion dramatically. A precise estimate of such a minimum molecular weight cannot be given; on the basis of a reptation mechanism one might expect quite a large molecular weight to be necessary.

During the shorter time scale, both the kinetic and the thermodynamic properties of the system are changed. The polymer solution appears to be more compatible with the nonsolvent. The thermodynamics during the shorter time scale are mainly determined by the enthalpic interaction of the polymeric additive with the nonsolvent. Other interactions do not deviate from the effects in the ternary situation without the PVP. Significant is that the interaction between the two polymers does not seem to have any effect, as long as the two polymers remain miscible.

Calculation of the initial composition paths indicates that when a polymeric additive is used, the polymer concentration in the skin layer remains low. The initial nonsolvent flux though the interface increases very much upon addition of the nonsolvent-soluble polymer.

It appears that the initial composition paths are not very sensitive to the values of the kinetic parameters.

An increase of the amount of solvent in the coagulation bath resulted in a reduction of the amount of polymer in the top layer until it becomes zero. This facilitates the transition to the behavior belonging to the longer time scale (i.e., demixing into two phases), since all diffusion processes are faster in more diluted solutions. Effectively, the addition of solvent to the coagulation bath will cause the polymer solution to demix quickly. This implies that even for very high solvent concentrations, delay of demixing is not possible. This is in accordance with practical experience [28].
The cloudpoint curves valid for the longer time scale are always situated close to the polymersolvent axis. During the shorter time scale, very instable compositions in the skin layer are created, according to the phase behavior of the longer time scale. One could say that the short time scale is equivalent to a very deep quench of the polymer solution. It may well initiate spinodal decomposition processes.

Addition of PVP suppresses delay of demixing, as shown by transmission experiments. Demixing starts earlier. PVP slows down the rate of decrease in transmittance whenever demixing has started. Additions of PVP K30 and PVP K90 show approximately analogous trends. The effects of PVP K90 are stronger, but equivalent to the effects of PVP K30.

The trends that are found are in accordance with the theoretical results.

Summarizing we can say that the introduction of a nonsolvent-soluble polymeric additive inhibits any delay of demixing, as long as the diffusion between the two polymers is significantly slower than the other diffusion processes taking place.

\section{List of symbols}

$c_{i} \quad$ concentration of component $i\left(\mathrm{~kg} \mathrm{~m}^{-3}\right)$

$g_{i j} \quad$ (concentration dependent) interaction parameter between components $i$ and $j$ $(-)$

$J_{i} \quad$ volume flux of component $i$ relative to component 3 (PES) $\left(\mathrm{m}^{3} \mathrm{~m}^{-2} \mathrm{~s}^{-1}\right)$

L matrix of phenomenological coefficients

$L_{i j} \quad$ phenomenological coefficient between components $i$ and $j$

$m$ spatial coordinate corrected for movement of the interface (m)

$M_{i} \quad$ molecular weight of component $i$ (g/ $\mathrm{mol}$ )

$s \quad \bar{v}_{1} M_{1} / \bar{v}_{2} M_{2}$

$r \quad \bar{v}_{1} M_{1} / \bar{v}_{3} \mathbf{M}_{3}$

$\mathbf{R} \quad$ inverse matrix of $L$ 
$\boldsymbol{R}_{i j} \quad$ friction coefficient between components $i$ and $j$

$t \quad \bar{v}_{1} M_{1} / \bar{v}_{4} M_{4}$, or time (s)

$u_{2} \quad \phi_{2} /\left(\phi_{2}+\phi_{1}\right)(-)$

$u_{4} \quad \phi_{4} /\left(\phi_{4}+\phi_{1}\right)(-)$

$v_{i} \quad$ velocity of component $i\left(\mathrm{~m} \mathrm{~s}^{-1}\right)$

$v_{2} \quad \phi_{2} /\left(\phi_{2}+\phi_{3}\right)(-)$

$u_{3} \quad \phi_{3} /\left(\phi_{3}+\phi_{4}\right)(-)$

$\bar{v}_{i} \quad$ specific volume of component $i\left(\mathrm{~m}^{3}\right.$ $\left.\mathrm{g}^{-1}\right)$

$w_{2} \quad \phi_{2} /\left(\phi_{2}+\phi_{4}\right)(-)$

$\mathbf{X}$ vector of driving forces (chemical potential gradients)

$x \quad$ spatial coordinate in the polymer solution (m)

$X(t) \quad$ distance between the interface on $t$ and the interface on $t=0(\mathrm{~m})$

$y \quad$ spatial coordinate in the coagulation bath (m)

$\mu_{i} \quad$ chemical potential of component $i$ ( $\mathrm{J}$ $\mathrm{mol}^{-1}$ )

$\phi_{i} \quad$ volume fraction of component $i(-)$

\section{References}

[1] ]S. Loeb and S. Sourirajan, Sea water demineralization by means of an osmotic membrane, Adv. Chem. Ser., 38 (1962) 117.

[2]H. Tompa, Polymer Solutions, Butterworths, London, 1956.

[3]C.C. Hsu and J.M. Prauznitz, Thermodynamics of polymer compatibility in ternary systems, Macromolecules, 7 (1974) 320.

[4]F.W. Altena and C.A. Smolders, Calculation of $1-1$ phase separation in a ternary system of a polymer in a mixture of solvent and nonsolvent, Macromolecules, 15 (1982) 1491.

[5]P.J. Flory, Principles ot Polymer Chemistry, Cornell University Press, New York, 1953.

[6]C. Cohen, G.B. Tanny and S. Prager, Diffusion-controlled formation of porous structures in ternary polymer solutions, J. Polym. Sci., Polym. Phys. Ed., 17 (1979) 477.

[7]A.J. Reuvers, J.W.A. van den Berg and C.A. Smolders, Formation of membranes by means of immersion precipitation. Part I. A model to describe mass transfer during immersion precipitation, J. Membrane Sci., 34 (1987) 45.

[8]A.J. Reuvers and C.A. Smolders, Formation of membranes by means of immersion precipitation. Part II. The mechanism of formation of membranes prepared from the system CA/acetone/water, J. Membrane Sci., 34 (1987) 67.

[9]C.A. Smolders, A.J. Reuvers, R.M Boom and I.M. Wienk, Microstructures in Membranes. Part 1. Macrovoid formation, J. Membrane Sci., 73 (1992) 259.

[10]L. Yilmaz and A.J. McHugh, Modelling of asymmetric membrane formation. I. Critique of evaporation models and development of a diffusion equation formalism for the quench period, J. Membrane Sci., 28 (1986) 287.

[11 ]C.S. Tsay and A.J. McHugh, Mass transfer modeling of asymmetric membrane formation by phase inversion, $\mathrm{J}$. Polym. Sci., Polym Phys. Ed., 28 (1990) 1327.

[12]C.S. Tsay and A.J. McHugh, Dynamics of the phase inversion process, lecture presented at the International Congress on Progress in Membrane Science and Technology (PMST), June 1991, University of Twente, Netherlands.

[13]J.A. van 't Hof, H.H.W. Rolevink, R.M. Boom and C.A. Smolders, Preparation of asymmetric gas separation membranes with high selectivity by a dual-bath coagulation method, J. Membrane Sci., 70 (1992) 17.

[14]P. Aptel, N. Abidine, F. Ivaldi and J.P. LaFaille, Polysulphone hollow fibers - effect of spinning conditions on ultrafiltration properties, J. Membrane Sci., 22 (1985) 199.

[15] L.Y. Lafrenière, F.D.F. Talbot, T. Matsuura and S. Sourirajan, Effect of polyvinylpyrrolidone additive on the performance of polyethersulfone ultrafiltration membranes, Ind Eng. Chem. Res., 26 (1987) 2385.

[16]T.A. Tweddle, O. Kutowy, W.L. Thayer and S. Sourirajan, Polysulfone ultrafiltration properties, Ind. Eng. Chem. Prod. Res. Dev., 22 (1983) 320.

[17]E. Roesink, Microfiltration: Membrane Development and Module Design, Ph.D. Thesis, University of Twente, Netherlands, 1989.

[18]I. Cabasso, E. Klein and J.K. Smith, Polysulphone hollow fibers. I. Spinning and properties, J. Appl. Polym. Sci., 20 (1976) 2377; II. Morphology, J. Appl. Polym. Sci., 21 (1977) 165.

[19]R.M Boom, I.M. Wienk, Th. van den Boomgaard and C.A. Smolders, Microstructures in Membrane. Part 2. The role of a polymeric additive, J. Membrane Sci., 73 (1992) 277.

[20] J.A. Wesselingh and R. Krishna, Mass Transfer, Ellis Horwood, New York, 1990.

[21]F. van Beckum, University of Twente, Netherlands, 1990 , private communication.

[22] Ph. Radovanovic, S.W. Thiel and S.-T. Hwang, Formation of asymmetric polysulfone membranes by immersion precipitation. I. Modelling mass transfer during gelation, J. Membrane Sci, 65 (1992) 213; II. The effects of casting solution and gelation bath compositions of membrane structure and skin formation, J. Membrane Sci., 65 (1992) 231.

[23]P.M. Dew and J.E. Walsh, A set of library routines for solving parabolic equations in one space variable, Assoc. Comput. Mach. Trans. Math. Software, 7 (1981) 295. 
[24]G. Tkacik and L. Zeman, Component mobility analysis in the membrane forming system water $/ N$-methyl-2pyrrolidone/polyethersulfone, J. Membrane Sci., 31 (1987) 273.

[25]L. Zeman and G. Tkacik, Thermodynamic analysis of the membrane forming system $\mathrm{H}_{2} \mathrm{O} / \mathrm{NMP} / \mathrm{PES}, \mathrm{J}$. Membrane Sci., 36 (1988) 119.

[26]R.M. Boom, R. Reinders, H.H.W. Rolevink, Th. van den Boomgaard and C.A. Smolders, Equilibrium thermodynamics of a quaternary membrane forming system with two polymers. 1. Calculations; 2. Experimental, Macromolecules, in press.

[27]P-G. De Gennes, Scaling Concepts in Polymer Physics, Cornell University Press, Ithaca, 1979.

[28]R.M Boom, Membrane Formation by Immersion Precipitation: The Role of a Polymeric Additive, Ph.D. Thesis, University Twente, Netherlands, 1992. 